\title{
La haecceitas como base de la solitudo en Duns Escoto
}

\author{
Haecceitas as Base of the Solitudo in Duns Scotus
}

GLORIA SILVANA ELÍAS*

\begin{abstract}
Resumen: El objetivo de este escrito es abordar la problemática filosófica del principio de individuación desde Duns Escoto, señalando las diferencias que al respecto sostuvo con la postura aristotélico-tomista, por un lado; por otro, mostrar cómo la pregunta por el concreto en la Antigüedad condujo en el Medioevo, a partir de la metafísica creacionista, a una reflexión cuyo término será inédito: la reflexión sobre el concepto de persona.

Palabras clave: Duns Escoto, Haecceitas, Solitudo.
\end{abstract}

\begin{abstract}
The aim of this paper is to address the problem of philosophical principle of individuation from Duns Scotus, noting the differences about the position he held with Aristotelian-Thomist, on one side; further show how the question of the concrete in antiquity led in the Middle Ages, from the creationist metaphysics, a term which is unprecedented reflection: reflection on the concept of person.
\end{abstract}

Keywords: Duns Scotus, Haecceitas, Solitudo.

\section{Introducción}

Duns Escoto, influenciado por Avicena, propuso su teoría de la natura communis y la haecceitas, entendida ésta como principio de individuación del compuesto, en franca oposición a la teoría aristotélico-tomista que postulaba la materia signata quantitate. En el caso del hombre, Duns entendió que la heceidad es condición para la existencia incomunicable de cada ser humano. La solitudo es, justamente, la estructura óntica, irrepetible e incomunicable de la persona ${ }^{1}$.

El objetivo de este escrito es abordar la problemática filosófica del principio de individuación desde Duns Escoto, señalando las diferencias que al respecto sostuvo con la postura aristotélico-tomista, por un lado; por otro, mostrar cómo la pregunta por el concreto en la

Fecha de recepción: 26/03/2013. Fecha de aceptación: 17/12/2013.

* Universidad Nacional de Jujuy. Facultad de Humanidades y Ciencias Sociales-CONICET (Argentina). Profesora Adjunta de Antropología Filosófica e Investigadora de Conicet. correo: gloriasilvanaelias@yahoo. com.ar. Las líneas de investigación que llevo adelante son "Duns Escoto: entre potentia Dei y libertad humana. Antecedentes y posición escotista" y "La contingencia como modalidad entitativa y operativa en la antropología de Duns Escoto. Proyecciones en la actualidad”, ambas financiadas por Conicet. Una de mis publicaciones más recientes es Duns Escoto: contingencia y posibilidad en la configuración de lo real, libro publicado en el 2013 por la Universidad Nacional de Tucumán-Argentina.

1 En esta línea reflexiona J. Merino: Juan Duns Escoto. Introducción a su pensamiento filosófico-teológico, BAC, Madrid, 2007, pp. 80-85. 
Antigüedad condujo en el Medioevo, a partir de la metafísica creacionista, a una reflexión cuyo término admitirá una mirada inédita: la reflexión sobre el concepto de persona.

\section{Materia y Principio de individuación en la perspectiva aristotélico-tomista. El planteo de Duns Escoto}

Como es sabido, el hilemorfismo aristotélico concibe en toda sustancia corpórea compuesta dos co-principios: materia y forma. La materia es vista como el receptáculo potencial de la forma, y ésta por su parte actualiza la materia, de modo tal que entre ambos principios conforman una sustancia. Para Aristóteles, la forma es principio de especificación, mientras que la materia es principio de individuación. No obstante, si bien la cosa singular es el sujeto de atribución de los accidentes, Joseph Moreau hace notar que en la predicación de la esencia, la función de sujeto último la tiene la materia²; por ello, Aristóteles se refiere a la materia del siguiente modo:

«Llamo materia a lo que por sí mismo no puede ser definido ni un algo ni un cuanto, ni ninguna de las demás categorías por las cuales se define el ente. Porque existe algo respecto del cual cada una de estas categorías se predica y cuyo ser es diferente del ser de cada una de las categorías, pues todas las categorías, a excepción de la ousía, son predicados de la ousía, siendo ésta en sí misma predicado de la materia. Entonces, el último sustrato en sí mismo no es algo, ni cuanto, ni nada semejante» ${ }^{3}$.

La materia no es ousía ${ }^{4}$ dice Aristóteles, en tanto que le falta ser un esto, un algo absolutamente determinado. No obstante, es un esto en potencia ¿Se infiere de ello que es por la forma por lo que la sustancia es un algo concreto? Por la forma o esencia se conoce la sustancia, y a la vez, en el ámbito de las sustancias sensibles, la forma no puede estar separada del individuo concreto ${ }^{5}$. Con todo, Aristóteles no identifica la forma con la sustancia individual, puesto que como bien sabemos, la forma «caballo» se extiende a todos los individuos que conforman la especie caballo; lo que hace que entre los individuos de la misma especie halla distinción son los accidentes, o para ser más precisos, es la materia. Ésta no produce diferencia específica sino numérica. Ahora bien, sobre este punto también hay distintas interpretaciones, puesto que en los capítulos 4 a 6 de Metafísica VII, Aristóteles deja planteado la identificación de la esencia con el sujeto particular. Este es el parecer de Enrico Berti, quien entiende que el principio de individuación para el Estagirita radica en la forma:

Pero la causa formal es fundamental, es importantísima para Aristóteles; el alma es la causa formal, él la llama también la causa del ser, la causa de la sustancialidad. Entonces, siendo la causa de la sustancialidad, la forma es la sustancia en el sentido

2 Cfr. J. Moreau: Aristóteles y su escuela, EUDEBA, Bs. AS., 1993, p. 93.

3 Aristóteles: Metafísica VII 3, 1029a 20-25. Traducción directa del griego, Introducción, Exposiciones sistemáticas e Índices de Hernán Zucchi, Ed. Sudamericana, Bs. As., 2000, p. 338.

4 Como es sabido, la ousía tiene diversas acepciones: sujeto individual (ousía primera), especie y género (ousía segunda), o quididad o esencia de la sustancia, por la cual la sustancia es cognoscible.

5 Cfr. Ibid., I 9, 991 b 1, p. 144. 
primero, es ousía proté. Entonces, se puede decir que, en el libro Zeta, Aristóteles ha determinado una de entre las causas primeras de los entes, a saber, la causa formal. Esta es la forma propia de cada sustancia, de cada sustancia individual...donde Aristóteles dice que la causa de Aquiles es Peleo, y la causa de ti es tu padre, dice también: yo tengo mi forma y tú tienes la tuya (1071 a 28-29). De aquí se deriva que la forma, tanto la mía como la tuya -cada uno tiene una-, es, consecuentemente individual. ${ }^{6}$

En esta línea, puede comprenderse el siguiente pasaje: «... pues hombre debe entenderse aquí como materia, pero la materia no produce diferencia [específica]; y por esta razón los ejemplares de hombre no son otras tantas especies de hombre, aunque sean otras las carnes y los huesos de que éste o aquél se constituyen» ${ }^{7}$. Ahora bien, una conclusión a la que se puede arribar es que, en el ámbito de las cosas sensibles, lo que produce la individuación es la materia:

Es evidente que el cielo es uno solo. Porque si hubiera tantos cielos como hay hombres, habría un principio que, en cuanto a la forma sería el mismo para cada uno, pero sería múltiple numéricamente. Mas, todo cuanto es múltiple según el número, tiene materia (pues en una pluralidad el discurso es uno y el mismo, como por ejemplo, es uno tanto para el hombre en general como para Sócrates). Pero el primer «lo que es ser esto» no tiene materia, porque es una entelequia; por tanto, el primer motor inmóvil es uno ${ }^{8}$.

El principio de individuación aristotélico ha recibido diversas interpretaciones en toda la Edad Media, puesto que cada filósofo postulaba su concepción respectiva. En el caso de Santo Tomás, éste entendió que la forma es efectivamente un principio de especificación, mientras que el principio de individuación provenía de la materia, mas no la materia indeterminada sino aquella que se le añade el concurso de la cantidad ${ }^{9}$. En De ente et essentia, Tomás explica justamente que la materia sola no es la esencia de la cosa, ya que ésta es cognoscible y ordenada en una especie y un género por la forma; tampoco es principio de conocimiento. No obstante, tampoco la forma por sí sola puede decirse la esencia de una sustancia compuesta: «Queda pues que el nombre de esencia en las sustancias compuestas significa aquello que es compuesto de materia y forma» ${ }^{10}$. Acto seguido, Tomás hace la siguiente precisión:

Pero puesto que el principio de individuación es la materia, acaso parezca que de esto se siga que la esencia -la cual a la materia en sí comprende simultáneamente

6 E. Berti, Estructura y significado de la Metafísica de Aristóteles, Buenos Aires, Oinos, 2011, p. 105.

7 Metafísica X 9, 1058b 6, p. 453. Lo que está corchetes es mío.

$8 \quad$ Ibid.XII 8, 1074a 30-35, p.530.

9 Respecto del principio de individuación en las distintas escuelas medievales puede consultarse M. Beuchot, «El principio de individuación en la Edad Media y su desembocadura en Leibniz», en: Aristas de la Filosofía Medieval, PPU, Barcelona, 1995, pp. 119-127.

10 Tomás de Aquino: De ente et essentia, traducción de Mons. Luis Lituma y Alberto de Reyna, Losada, Bs. As., 2003 , p. 18. 
con la forma- sea particular solamente y no universal. De aquí se seguiría que los universales no tendrían definición, si la esencia es aquello que se significa por la definición. Y por esto hay que saber que la materia tomada no en cualquier modo es el principio de individuación, sino sólo la materia signada ${ }^{11}$.

La materia signata quantitate significa aquella materia considerada bajo determinadas dimensiones, la cual a su vez no es la que forma parte de la definición de «hombre», sino la que hace que Sócrates, que es hombre, sea un hombre concreto. Como explica Beuchot, la cantidad concurre a individualizar junto con la materia en el compuesto, la cantidad adviene al compuesto en tanto accidente y por virtud de la forma. Es decir, la forma en tanto principio de especificación permite la inherencia de la cantidad en la sustancia sensible, en tanto que los accidentes de la sustancia le vienen por virtud de su forma, incluso la cantidad, que está en la línea de la materia ${ }^{12}$. En Suma contra los gentiles, Tomás explica que las quididades de los géneros y especies se individualizan por la materia determinada de tal o cual individuo, aun cuando su quididad contenga la forma y la materia en general ${ }^{13}$.

En suma, la materia determinada por la cantidad es principio de individuación de la sustancia corpórea ${ }^{14}$.

En Opus Oxoniense, Duns Escoto se pregunta si en los seres sometidos a generación y corrupción, existe una entidad positiva ${ }^{15}$, que posea un ser propio y que sea distinta realmente de la forma. Duns así lo cree, de lo contrario no se podría hablar de compuesto en el caso de la sustancia corpórea. Si bien Escoto entiende que la materia existe por sí, con todo, hay diversas opiniones al respecto. En la distinción referida, éste va señalando dichas opiniones, a saber: algunos consideran que la materia sería todo, y no sólo una parte; la materia es considerada intrínseca a la forma ${ }^{16}$. A esta postura Escoto responde que se necesitan dos elementos que compongan un concreto para que pueda haber en éste generación o corrupción, y que además el paso de un contrario a otro se da en la materia. Ahora bien, la materia es una potencia, pero Escoto sostiene que la materia es por una parte, potencia objetiva, y por otra, potencia subjetiva. Éste define la potencia objetiva como: «La potencia al ser propio, ser simpliciter de aquello que está en potencia; la potencia de alguna esencia substancial o accidental al propio ser, fundada en esa esencia que es su propio ser» ${ }^{17}$. Dicho de otra manera, la materia en tanto que potencia objetiva es la capacidad que algo tiene de ser ya de un modo ya de otro, incluyendo la capacidad primigenia, a saber, la potencia a su ser

11 Ibid., p. 20.

12 Cfr. Beuchot: op. cit., pp. 120-121.

13 Aquino: Suma contra los gentiles, I, c. 21, tomo I, segunda edición dirigida por los Padres Carcedo O.P. y Robles Sierra O. P. Introducción de José De Garganta, O.P., BAC, Madrid, 1967, p. 155.

14 Respecto de las interpretaciones de la fórmula tomista materia signata quantitate puede consultarse A. G. Fuente, «Interpretaciones tomistas de la fórmula 'materia signata quantitate'», Estudios Filosóficos, 10 (1961), pp. 461-470.

15 Cfr. Opus Oxoniense II, d.12, q.1: utrum in substantia generabili et corruptibili sit aliqua entitas positiva realiter distincta a forma, ed. Fernández García, tomo II, Quaracchi, 1914, pp. 497- 542.

16 Cfr. Ibid., n.3, pp. 498-499. Gilson hace notar que esta posición bien puede ser la de Alejandro de Afrodisia, ver E. Gilson: Juan Duns Escoto. Introducción a sus posiciones fundamentales, EUNSA, Navarra, 2007, p. 428.

17 Escoto: Quaestiones subtilissimae in Metaphysicam Aristotelis, IX, qq. I-XV, ed. Vivès, tomo IV, p.763: «Quadam ad esse proprium, et simpliciter illius, quod est in potentia, est cuiuscunque essentiae substantialis, vel accidentalis ad proprium esse, et fundatur in illa essentia, cuius est illud proprium esse». 
primero y fundamental; es la potencialidad inherente a una esencia en lo que respecta a sus diversos actos intrínsecos y propios, empezando por la potencialidad a su acto primero de ser y terminando por los actos accidentales. En este punto, Escoto pone por ejemplo la blancura (en el orden accidental), y plantea: ésta puede estar en una pared si es pintada, actualizándose de ese modo, y este «poder blanquear» es anterior al acto mismo de blanquear, y al acto mismo de ser blancura de la pared, «es la potencial blancura antes propiamente de que haya blancura ${ }^{18}$. La potencia objetiva, por tanto, es la potencia al acto intrínseco y propio del ente, a saber: su existencia, ya sea de la sustancia misma, ya sea del accidente.

Ahora bien, Escoto distingue dos tipos de potencia objetiva ${ }^{19}$ :

a) potencia objetiva al acto puro: es la potencia objetiva al acto de existir que es total y simultáneo (actum simul totum), se refiere a la esencia de los entes permanentes;

b) potencia objetiva al acto mezclado con potencia: se trata de la potencia objetiva a un acto de ser sucesivo. Como bien lo describe Miralbell: «se trata de la potencia a un acto propio de existir en el que junto al acto que es término de la potencia, simultáneamente persiste una potencialidad a un acto posterior, que es lo que ocurre a los entes que existen según sucesión, en los cuales la potencia esencial nunca alcanza en simultaneidad y en totalidad el acto al que se ordena» ${ }^{20}$.

Con respecto a la potencia subjetiva, Escoto la define como potencia al ser secundum quid, no propio, como potencia al ser extrínseco, al ser-como-si (quasi) y al ser en cierto modo (secundum quid) de aquello mismo que es lo que está en potencia a tal ser. Esta potencia sólo se da en aquel ente que puede recibir el ser de otro ente, siendo sujeto de un acto, por ejemplo, la pared tiene potencia subjetiva a ser blanqueada, a poder recibir de otro la actualización de ser blanqueada. Como se ve, atribuirle a la materia una función de sujeto implica reconocerle una realidad propia. Además, como el mismo Estagirita señala, la materia cumple una serie de funciones que suponen de ella una cierta realidad: es naturaleza, y por tanto, cierto principio o causa del movimiento y reposo del ente, es sujeto de las mutaciones sustanciales, etc. Ello lleva a sostener a Escoto que la materia no recibe el esse de su forma, sino que tiene el suyo propio ${ }^{21}$. El argumento central de Escoto es que lo engendrable se compone de materia y forma, pero si se reduce la materia a nada, es compuesto de forma y nada, lo cual es absurdo. De ello se concluye: «La materia tiene una cierta realidad positiva fuera del intelecto y de su causa, y es en virtud de esta realidad que ella puede recibir las formas sustanciales, que son simplemente actos» ${ }^{22}$. La materia es un ser absoluto distinto y anterior a la forma, por lo que puede existir sin la forma sustancial o accidental.

18 Cfr. Ibid.

19 Cfr. Ibid.: «Sed divititur illa potentia in potentiam, quae est ad actum purum: et quae ad actum mixtum potentiae. Secundum enim diversitatem effentiarum potentia est ad actum diversimode recipiendum, quandoque simul totum, sicut essentiae permanentium natae sunt recipere esse; quandoque successive, ita quod semper cum actu terminante potentiam, stet potentia ad viteriorem actum: sicut in successivis, ita quod ibi nunquam potentiae simul succedit actus oppositus».

20 I. Miralbell: El dinamicismo voluntarista de Duns Escoto. Una transformación del aristotelismo, EUNSA, Pamplona, 1994, p. 53.

21 Cfr. Q. in Met. VIII, q.3, n.5.

22 Ox. II, d.12, q.1, n.11, p. 504. 
Ahora bien, la materia no puede ser ella misma su principio de individuación (en tanto existente), por tanto, Escoto ve necesario inquirir cuál sea el principio de individuación. Sobre el principio de individuación de Escoto Gérard Sondag ha escrito una exégesis detallada del texto en el que aquél desarrolla su tesis, y ha traducido los pasajes claves ${ }^{23}$. Como analizar el texto en su totalidad excede el propósito de este escrito, presentaremos sólo el núcleo de su planteo. Escoto desarrolla el mismo en el segundo libro de Ordinatio, dedicado a la creación. En éste hay varias cuestiones dedicadas a los ángeles. La distinción tercera, en su primera parte, se titula De principio individuationis, aun cuando el mismo no haya sido el que Escoto le dio ${ }^{24}$. Lo que le interesa a éste es saber en qué reside la distinción de ángeles en personas. No obstante, entiende que para poder dar respuesta a esta cuestión debe previamente inquirir sobre aquello que distingue las sustancias materiales en individuos. Este periplo está mostrando que en el presente estado el intelecto creado conoce a partir de las cosas materiales, y desde éstas inicia el proceso cognoscitivo de las sustancias inmateriales, lo que hace suponer -según Sondag- que el principio de individuación es uno y el mismo en las naturalezas creadas consideradas (sustancias materiales y los ángeles), el cual no reside ni en la materia ni en la cantidad ${ }^{25}$.

A lo largo de las cuestiones se van enunciando opiniones diversas sobre el tema, a las que Escoto niega argumentativamente. Sólo vamos a referirnos a algunas. Por ejemplo: a aquellos que creen que la materia es naturalmente individual Escoto aduce que de ser así, nuestro intelecto concibe las cosas contrariamente a su naturaleza, en cuanto que las concibe en sus aspectos universales. Escoto postula que entre la unidad real del singular y el universal, existe una unidad menor que la numérica, que depende de la natura communis ${ }^{26}$, la cual a su vez es indiferente a la universalidad como a la unidad del singular ${ }^{27}$. Por tanto, no se sostiene la tesis que la sustancia material sea individual por pleno derecho, más bien hay que pensar en algún elemento positivo intrínseco ${ }^{28}$; Escoto entiende que la especie y el género forman la quididad del ente, por ejemplo, animal racional, pero el orden de la individualidad (que como se vio no se da en orden de la materia) tampoco se da en el de la quididad, puesto que ésta más bien es principio de especificación: «la entidad individual se da en un orden diverso al de la quididad», excepto el ser infinito, ninguna quididad contiene aquello que le permite ser haec ¿Cómo supera Duns el orden de lo común para entrar en el orden de lo individual? A lo largo de las cuestiones habrá de negar que sea por vía de

23 G. Sondag: Duns Scot. Le principie d'individuation. Texte latin en vis-à-vis. Introduction, traduction et notes per Sondag, Vrin, París, 2005.

24 Cfr. Ordinatio II, ed. Vat., 1973.

25 Sondag: op. cit., p. 13.

26 Cfr. Ord. II, d. 3, p. 1, q. 1, n. 3, p. 396.

27 Cfr. Ibid., n.7: «Aliqua est unitas in re realis, absque omni opeatione intellectus, minor unitate numerali sive unitate propria singularis, quae unitas est naturae secundum se; et secundum istam unitatem propriam naturae, ut natura est, natura est indifferens ad unitatem singularem; non ergo de se est sic una unitate illa, scilicet unitate singularitatis».

28 Cfr. Ibid, q. 2, n. 4, pp. 416-417: «Concedo igitur conclusiones istarum rationum, quod necesse est per aliquid positivum intrinsecum huic lapidi, tamquam per rationem propiam, repugnare sibi dividi in partes subiectivas; et illud positivum erit illud quod dicetur esse per se causa individuationis, quia per individuationem intelligo illam individibilitatem sive repugnantiam ad divisibilitatem». 
negación, negará también la individuación por la existencia, o por la cantidad, para llegar finalmente a postular la haecceitas como ultima realitas entis:

Por tanto, 'esta entidad' no es materia o forma o compuesto, en cuanto cualquiera de ellos es 'naturaleza', sino que es la realidad última del ente que es materia o <del ente $>$ que es forma, o < del ente $>$ que es compuesto. De modo que todo lo que es común, y, sin embargo, determinable, todavía puede ser distinguido (por más que sea una única cosa) en realidades múltiples formalmente distintas, de las cuales ésta no es formalmente aquélla; y ésta es formalmente la entidad de la singularidad y aquélla la entidad de la naturaleza formalmente. Y no pueden estas dos realidades ser cosa y cosa, tal como pueden serlo la realidad de la que se toma el género y la realidad de la que se toma la diferencia (a partir de las cuales se toma la realidad específica), sino que siempre en lo mismo (sea en parte, sea en todo) son realidades de la misma cosa, formalmente distintas ${ }^{29}$.

Como señala Gilson, se trata de una individuación de la quididad pero no por la quididad, es una individuación de la forma, pero no por la forma ${ }^{30}$. La individuación permite la determinación completa del singular, sin apelar a la existencia, es el perfeccionamiento definitivo de la forma sustancial, por la cual un ente es un esto concreto e irrepetible. La heceidad, en suma, es la última actualidad de la forma. Además, es importante tener presente que para Escoto, el singular se compone al menos de seis entidades: la materia universal y la materia particular, la forma universal y la forma individuada, el compuesto universal y el compuesto individuado ${ }^{31}$, pero lo individual adquiere primacía sobre el universal, y la heceidad es la actualización definitiva de la forma, de la materia y del compuesto. Ésta no se conoce directamente, sino por mediación de conceptos abstractos ${ }^{32}$.

De lo visto hasta acá, podemos concluir que la entidad específica y la entidad individual difieren porque la primera es divisible en partes cuantitativas, mientras que la segunda no. Además, la diferencia específica es una forma, en tanto que la heceidad es la actualidad última de la forma.

\section{La solitudo y su relación con el concepto de persona}

La especulación sobre el concepto persona ha sido promovida a partir de la confesión de un Dios Trino, y la confesión de que Jesús era hombre y Dios en unidad de persona, y

29 Cfr. Ibid., q. 6, n. 15, p. 484: «Non est igitur 'ista entitas' materia vel forma vel compositum, in quantum quodlibet istorum est 'natura', - sed est ultima realitas entis quod est materia vel quod est forma vel quod est compositum; ita quod quodcumque commune, et tamen determinabile, adhuc potest distingui (quantumcumque sit una res) in plures realitates formaliter distinctas, quarum haec formaliter non est illa: et haec est formaliter entitas singularitatis, et illa est entitas naturae formaliter. Nec possunt istae duae realitates esse res et res, sicut possunt esse realitas unde accipitur genus et realitas unde accipitur differentia (ex quibus realitas specifica accipitur), - sed semper in eodem (sive in parte sive in toto) sunt realitates eiusdem rei, formaliter distinctae».

30 Cfr. Gilson: op. cit., p. 459.

31 Para un análisis detallado de esto ver Sondag: op. cit., pp. 57-58.

32 Sabido es que el término haecceitas no figura en Ordinatio, pero sí en Rep. Par. II, d. 12, q. 6, nn. 8, 13. En esta última obra, ya intervinieron los discípulos de Escoto, por lo que se cree que el término no sea del mismo Duns. 
en este contexto teológico se enmarca la reflexión sobre el ser personal. A continuación, reflexionaremos sobre dicho ser en la perspectiva escotista, pero centrándonos en su concepción más bien filosófica, y no tanto teológica. Previo a Escoto, ya se habían configurado al menos en la Escolástica tres vías de determinación del concepto persona: 1) la de San Agustín, quien aprehende la persona en el horizonte de una relación; 2) la de Boecio $^{33}$, que la define como una sustancia individual de naturaleza racional; 3) la de Ricardo de San Víctor $^{34}$, que la define como existencia incomunicable de naturaleza espiritual. Es a ésta a la que adhiere Escoto, porque refleja el estatuto existencial del hombre. Dice: «Tomo la definición que da Ricardo [...] cuya definición expone y corrige la definición de Boecio, que dice que la persona es sustancia individual de naturaleza racional; porque ésta implicaría que el alma es persona, lo que es falso» ${ }^{35}$. Pretende hallar un concepto unívoco, aplicable no sólo al hombre, sino también a Dios y a los ángeles. Al respecto Isidoro Manzano dedica una gran parte de sus escritos a tratar sobre el problema del ser personal. Su análisis demuestra que la entidad individuante ultimada por la heceidad no puede constituir la persona, pero tampoco la persona constituye el carácter espiritual de una sustancia; la naturaleza racional común del hombre (natura communis), y su individualidad a partir de la haecceitas, no es aún persona ${ }^{36}$. Dicho de otra manera, la entidad singular, suposito, hipóstasis o heceidad, es la perfección última en el orden ontológico, pero ser una naturaleza «haec» no es aún el ser personal, sino más bien condición de posibilidad. Además, la existencia se halla en un orden diverso que el ontológico, puesto que se refiere más bien a una causa que pone algo fuera de sí37. Dice Escoto: «Digo que la última distinción en el orden predicamental es la distinción individual y ésta es el último acto que pertenece «per se» a la coordinación predicamental, pero a esta distinción no pertenece la existencia actual: la existencia actual es, en efecto, acto último, pero posterior a toda coordinación predicamental» ${ }^{38}$. O sea, como señala Manzano, la persona supone el orden óntico-existencial, pero da lugar a un orden distinto, el orden personal ${ }^{39}$. Como consecuencia de esta postura, Escoto entiende que debemos pensar la persona sin ontologizarla ni entificarla, fundamentalmente porque toda naturaleza común es comunicable, mientras que la persona es incomunicable. La persona es el término de la naturaleza espiritual, siendo absoluta e independiente. Decimos además que la persona es incomunicable porque es única e irrepetible, en tanto no está en el orden óntico, por lo que no admite una aptitud a comunicarse como sí la tiene la materia y la forma. En efecto, Duns Escoto comprende a la persona como ultima solitudo, libre de cualquier dependencia real o

33 Cfr. Boecio: De duabus naturis, cap. 3.

34 Cfr. R. San Víctor De: De Trinitate IV, cap. 22.

35 Ord. I, d. 23, n.15, tomo V, ed. Vat., 1959.Otros lugares en los cuales Escoto trata sobre «persona» son: Ord. I, dd. 25, 26, 28; Ord. III, d. 1, Quodl. XIX.

36 Cfr. I. Manzano, «Ontología de la persona humana según Escoto», Antonianum (Roma) annus LXXVIII, Aprilis-Iunius 2003, fasc. 2, Roma, p. 327.

37 Vale puntualizar que en Escoto entre la esencia y la existencia no cabe una distinción real, tampoco de razón, sino ex natura rei.

38 Ord. II, ed. Vat., tomo VII, p. 421: «Dico quod ultima distinctio in coordintione praedicamentali est distinctio individualis et illa est ultimum actum, per se pertinentem ad coordinationem praedicamentalem; sed ad hanc non per se pertinet existentia actualis; existentia autem actualis est ultimus actus, sed posterior tota coordinatione praedicamentali».

39 Cfr. Manzano: op. cit., p. 330. 
derivada del ser con respecto a otra persona ${ }^{40}$; hace referencia a una estructura configuradora de la persona humana, que no debe entenderse como la soledad que acecha a la persona, sino como la estructura fundante de mi mundo personal y propio; el orden personal es el más radical y originario, «al principio», está la persona dice Manzano, y lo demás es personal por naturaleza ${ }^{41}$. Cada persona es absolutamente diferente, sin poder reducirse a otra persona; es un evento absoluto independiente e incomunicable que por su virtud una naturaleza singular individual queda determinada como personal.

Ahora bien, Escoto lleva a cabo una distinción entre la persona divina y la persona humana, refiriéndose a que la primera no puede absolutamente no comunicarse ni ser substituida ${ }^{42}$, en cambio, la persona humana es persona de modo tal que podría haber sido personada por otra persona distinta que por la que lo fue. Dice: «la naturaleza creada, por el contrario, aunque subsista en sí, no tiene nada intrínseco que imposibilite su dependencia. Por eso, sólo la persona divina tiene personalidad propia completa. La naturaleza creada personalizada en sí no la tiene» ${ }^{43}$.

En suma, la noción de persona instaura un orden trascendental, en el que cada individuo persona es irrepetible, independiente e incomunicable. Más allá de la diferencia entre la persona divina y la persona humana en Escoto, que no ha sido abordado aquí, se puede concluir que la persona establece una ultimidad más radical que la ultimidad de la heceidad que es ultima solitudo, irreemplazable es su condición de tal, persona que significa una existencia incomunicable, que no puede trasvasarse, ni intercambiarse. En suma, la naturaleza espiritual individuada es condición de posibilidad de la persona, que no significa por ello que sea anterior a la misma. El ser persona nos pone en el mundo como seres humanos cuya tarea de ex-sistir no podemos evadir, es decir, en ella no podemos ser reemplazados por otros.

\section{Bibliografía}

Aquino, Tomás de: De ente et essentia, traducción de Mons. Luis Lituma y Alberto de Reyna, Losada, Bs. As., 2003.

- : Suma contra los gentiles, I, tomo I, segunda edición dirigida por los Padres Carcedo O.P. y Robles Sierra O. P. Introducción de José De Garganta, O.P., BAC, Madrid, 1967.

Aristóteles: Metafísica VII 3, 1029a 20-25. Traducción directa del griego, Introducción, Exposiciones sistemáticas e Índices de Hernán Zucchi, Ed. Sudamericana, Bs. As., 2000.

Berti, Enrico: Estructura y significado de la Metafísica de Aristóteles. Traducción del italiano por Horacio Gianneschi, Oinos, Buenos Aires, 2011.

Beuchot, Mauricio: «El principio de individuación en la Edad Media y su desembocadura en Leibniz», en Aristas de la Filosofía Medieval, PPU, Barcelona, 1995, pp. 119-127.

Fuente, A. G., «Interpretaciones tomistas de la fórmula 'materia signata quantitate'», Estudios Filosóficos, 10 (1961), pp. 461-470.

Moreau, Joseph: Aristóteles y su escuela, EUDEBA, Bs. AS., 1993.

40 Cfr. Ord. III, d. 1, p. 1, q. 1: utrum possibili fuerit humanam naturam uniri Verbo in unitate suppositi, n. 66, p. 32, ed. Vat., tomo IX, 2006.

41 Cfr. Manzano: op. cit., p. 348.

42 Cfr. Quodl. XIX, BAC, Madrid, 1968, p. 690.

43 Ibid., n. 69, p. 690. 
Duns Scotus: Ordinatio I, tomo V, ed. Vat., 1959.

—: Opus Oxoniense II, ed. Fernández García, tomo II, Quaracchi, 1914.

—: Ordinatio II, ed. Vat., tomo VII, 1973.

—: Ordinatio III, ed. Vat., tomo IX, 2006.

-: Quaestiones subtilissimae in Metaphysicam Aristotelis, IX, qq. I-XV, ed. Vivès, tomo IV, 1894.

-: Quaestiones Quodlibetales XIX, BAC, Madrid, 1968.

Manzano, Isidoro: «Ontología de la persona humana según Escoto», Antonianum, (Roma), annus LXXVIII, Aprilis-Iunius 2003, fasc. 2.

Merino, José: Juan Duns Escoto. Introducción a su pensamiento filosófico-teológico, BAC, Madrid, 2007.

Miralbell, Ignacio: El dinamicismo voluntarista de Duns Escoto. Una transformación del aristotelismo, EUNSA, Pamplona. 1994.

Sondag, Gerard: Duns Scot. Le principie d'individuation. Texte latin en vis-à-vis. Introduction, traduction et notes per Sondag, Vrin, París, 2005. 